\title{
Electrical Variable Transmission for Hybrid Off-highway Vehicles
}

Thomas Vandenhove, Ahmed A-E. Abdallh, member, IEEE, Florian Verbelen, Mats Vande Cavey, and Jeroen Stuyts

\begin{abstract}
The push for less emissions has driven transportation towards electrification. The electrical variable transmission is a promising emerging component that has proven to be successful in passenger vehicles and is being considered in this paper for off-highway vehicles. By electromagnetically coupling the internal combustion engine with the wheels, allowing independent rotation, the engine is kept in its optimal operating range. This paper benchmarks the electrical variable transmission to one of the most successful hybrid topologies: the Toyota hybrid system. Flanders Make's Hybrid Electric Drivetrain CoDesign framework is being used to ensure optimal control decisions for both. Results show that the electrical variable transmission may reduce fuel consumption by $30 \%$ and total cost of ownership by $10 \%$.
\end{abstract}

Index Terms-Electric variable transmission, dynamic programming, energy management, off-highway vehicles, total cost of ownership.

\section{INTRODUCTION}

$\mathbf{T}$ he electrification of transportation is one of the most important factors to reduce global warming. Traditional single mechanical port electric machines, such as induction and permanent magnet machines, are intensively used by the automotive industry to create full or Hybrid Electric Vehicles (HEV) [1]. These traditional electric machines often require an additional mechanical device, e.g. a gearbox or planetary gear, to match the vehicle requirements or interface the different components. Therefore, an Electric Variable Transmission (EVT), which is a dual mechanical ports electric machine, was developed to reduce the use of such components [2].

An EVT is a competitive power split device that combines the functionality of two electric machines and a planetary gear set into one single electromechanical device [3]. An EVT has two mechanical ports and two electric ports. A decade of research has shown both the feasibility and reduced fuel consumption of an EVT in a HEV drivetrain [4], [5], [6], [7], [8]. These findings have been recently confirmed using more

This work was carried out for the EMTechno project (project ID: IWT150513) supported by VLAIO and Flanders Make, the strategic research center for the manufacturing industry.

T. Vandenhove, F. Verbelen, M. Vande Cavey and J. Stuyts are with Flanders Make, Belgium (e-mail: first-name.last-name@flandersmake.be), corresponding author: jeroen.stuyts@flandersmake.be

A. A-E. Abdallh is with ArcelorMittal Global R\&D Gent, OCAS N.V., J.Kennedylaan 3, 9060 Zelzate, Belgium (e-mail: ahmed.abdallh@arcelormittal.com; ahmed.abdallh@ieee.org)

F. Verbelen is also with the Department of Electromechanical, systems and metal engineering, Ghent University, Belgium (e-mail: florian.verbelen@ugent.be) sophisticated EVT models in [3] and [9]. However, to the best of the authors' knowledge, there is few research that studies the behavior of the EVT in off-highway (OHW) vehicles (e.g. tractors, forklifts, reach stackers ... ) [10], [11].

This paper aims to characterize the benefits of introducing an EVT system in OHW vehicles, such as lower fuel consumption, better power flow management and reduced battery ageing. The well-known Toyota Hybrid System (THS) (often called Hybrid Synergy Drive) [12] has been selected as a benchmark case. Both topologies are modeled in the Matlab embedded Hybrid Electric Drivetrain CoDesign framework developed at Flanders Make [13]. Here, Dynamic Programming (DP) is used to define the optimal set of inputs on the complete driving cycle in order to minimize, in this case, the fuel consumption.

The performance of each hybrid topology is evaluated using the same driving cycle. The framework allows the users to develop a topology (components, links, driving cycle), to define and solve the DP problem (states, inputs, cost function), to run the outputs of the DP as forward simulation and to post-process the data (total cost of ownership (TCO) analysis, visualization, efficiency analysis, etc.).

The paper is organized as follows: Sec II briefly introduces the EVT. Sec III describes the used methodology, including the DP framework as well as models of all considered components. Sec IV comprehensively presents and discusses the results of this analysis. Finally, $\mathrm{Sec} V \mathrm{~V}$ summarizes the conclusions.

\section{Electrical VARIABle Transmission}

During the past decade, in which fuel consumption and emissions have become key performance indicators, the EVT has gained a lot of interest to optimize fuel consumption [14]. Of the many different EVT topologies, a permanent magnet version, as described in [15] is used in this paper. An EVT differentiates itself from other hybrid systems and power split transmissions by offering two electromagnetically coupled rotors which are mechanically uncoupled. This creates two mechanical and two electrical ports within one machine.

The cross section of the considered EVT is shown in Fig 1. Both the inner rotor and stator are equipped with a distributed three-phase winding. The outer rotor consists of a single layer of permanent magnets and a DC-field winding, which are separated by a flux bridge. The purpose of this DC-field winding was introduced and explained in [15]. 


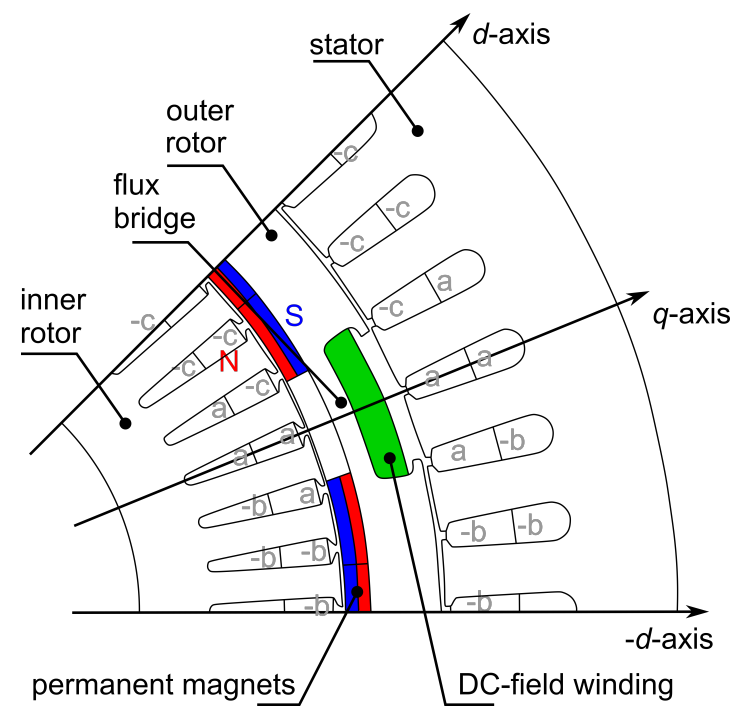

Fig. 1. Cross section of the EVT

TABLE I

MACHINE PARAMETERS OF THE CONSIDERED, UNSCALED, EVT.

\begin{tabular}{l|c|c|c}
\hline & Stator & Outer rotor & Inner rotor \\
\hline Rated mechanical power [kW] & - & 120 & 75 \\
Number of slots $N_{i}$ & 48 & - & 48 \\
Number of pole pairs $N_{p}$ & 4 & 4 & 4 \\
Outer radius [mm] & 175 & 123.5 & 102 \\
Inner radius [mm] & 124.5 & 103 & 57 \\
PM thickness $L_{m}[\mathrm{~mm}]$ & - & 5 & - \\
Active axial length $L[\mathrm{~mm}]$ & 87 & 87 & 87 \\
Width flux bridge [mm] & - & 5 & - \\
\hline
\end{tabular}

The shafts of the inner and outer rotor form the two mechanical ports while the windings of the inner rotor and stator form the two electrical ports. Slip rings are used to connect the inner rotor and DC-field winding. For clarity, the most important EVT parameters are shown in Table II.

\section{Methodology}

\section{A. Dynamic Programming}

Dynamic Programming is a global optimal control algorithm used to find the control inputs $u$ that minimize the total fuel consumption $F$ for state $x$ for steps $k=1 \rightarrow K$ :

$$
\begin{array}{ll}
\underset{u}{\operatorname{minimize}} & \sum_{k=1}^{K} F(k) \\
\text { subject to } & {\left[x_{k+1}, F(k)\right]=\mathcal{F}\left(x_{k}, u_{k}, k\right)} \\
& S o C(1)=\operatorname{SoC}(K) \\
& \text { drivingcycle }(k)
\end{array}
$$

As opposed to other (analytical) optimization methods which relate to the Potriagin minimal principle [16] to derive the necessary conditions for optimality [17], DP is a numerical optimization technique which uses the principle of optimality to derive the necessary conditions for optimality [18]. The DP algorithm examines all the feasible state driving cycle candidates that satisfy the necessary condition, by breaking down the global problem into local subproblems for every (reachable) discrete state and time instant. Due to its numerical framework, it is very suitable to describe discrete dynamics, nonlinear characteristics, and non-convex constraints, while global optimality is guaranteed when neglecting approximation errors introduced by mathematical modeling and numerical quantization [19]. When DP is used for continuous dynamics, e.g. battery state of charge (SoC), that state and its related control input need to be quantized into a finite number of options, creating an inherent trade-off between accuracy of the optimum and computing effort.

In the framework [13] an effort was done to guarantee fast and sufficiently accurate calculation of the optimum. First, all model evaluations in DP are performed using efficient stacking of matrix calculations such that the number of calculations is kept to a minimum. Then, as DP evaluates the same subproblem with a different value for the driving cycle at each timestep, it is possible to separate the DP evaluations which are time independent, i.e. the effect of the control input grid on the state is not dependent on the driving cycle. The time independent part is automatically extracted, evaluated just once and stored as a lookup table. All evaluations concerning states should preferably be in the time-independent part. This is often possible by a deliberate choice of mathematical control input to isolate a state, e.g. SoC state using $\Delta \mathrm{SoC}$ as input. With matching state and input, it is also recommended to match their quantization to avoid state leakage due to round-off errors. Given all speed ups, DP is able to accurately and efficiently evaluate both EVT and THS drivetrain topologies.

\section{B. Studied vehicle}

The Doosan D90s-7 forklift [20] has been selected as the reference $\mathrm{OHW}$ application as its power requirements and weight are within a sensible range from the reference EVT and THS models, which were originally developed for passenger car applications. This ensures that both topologies are suitable and sufficiently scalable. The details of the Doosan D90s-7 are shown in Table III. For modelling purposes, the weight of the components has been taken into account, as it affects the fuel consumption, but no feasibility analysis has been performed to ensure that these components would physically fit in the forklift.

\section{Modelling of the THS}

The THS topology is based on two electromotors (EM1 and EM2). A planetary gear system (PGS) splits the incoming power from the internal combustion engine (ICE) and transmits it to the rotor of EM1 and the rotor of EM2, of which the latter is also connected to the gearbox (GB) which drives the wheels. The GB is required to limit the torque peaks to the THS. The battery connects to the converters of EM1 and EM2 via a DC/DC converter and common DC-bus. Fig. 2 outlines the conceptual design of the THS topology.

The ratings of the ICE, EM1, EM2 and PGS are based on the Toyota Prius II [12]. As the forklift is ten times heavier 


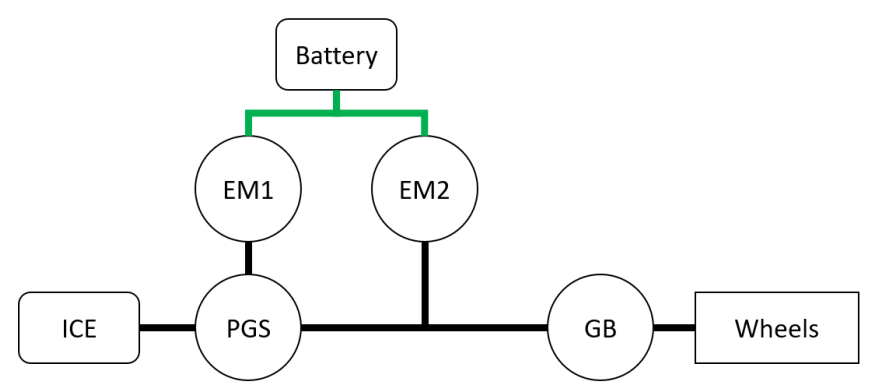

Fig. 2. THS topology (power converters not shown).

TABLE II

POWER RATINGS FOR THS TOPOLOGY [12], 20].

\begin{tabular}{l|c|c}
\hline & Toyota Prius II & Doosan D90s-7 \\
\hline Mass [T] & 1360 & 12275 (Diesel, unloaded) \\
ICE Power [kW] & 57 & 100 \\
EM1 Power [kW] & 50 & 250 \\
EM2 Power [kW] & 30 & 30 \\
PGS ratio (rps) & $2.6(78 / 23 / 30)$ & $2.6(78 / 23 / 30)$ \\
\hline
\end{tabular}

than the Prius II, the ICE power rating is doubled and the EM1 rating is multiplied by a factor 5 to cope with the mass difference. EM2 is kept at the same rating because it is sufficient for the chosen driving cycle. The PGS ratios are sufficient. Table I details these values for both vehicles. For reference, the Diesel Doosan D90s-7 engine has a peak power of $80.9 \mathrm{~kW}$ [20]. The mass increase due to hybridization is $664 \mathrm{~kg}$ for the EVT and $622 \mathrm{~kg}$ for the THS.

\section{Modelling of the EVT}

The inner rotor (rotor 1) of the EVT is attached to the ICE and the outer rotor (rotor 2) is connected to the GB of the vehicle, again to limit torque. The converters of stator and inner rotor (connected via the slip rings) are again connected to a common DC-bus, which is also connected to the battery unit via a DC/DC converter. Fig. 3 outlines the conceptual design of the EVT topology.

The EVT is programmed in the toolbox as a Look-Up Table (LUT) to speed up the DP calculation. This LUT outputs losses for any given set of inputs that is in range and was generated from the validated model of [15], [21], which in turn was based on a prototype, experimental tests and finite element calculations. That prototype was made for an average size passenger car, of which the size and torque requirements do not match the considered OHW vehicle (see also Table I To avoid a computational expensive redesign of the EVT, scaling laws for the EVT have been developed and validated [22]. This allows for a fast assessment of design modifications via axial $K_{a}$ and radial scaling $K_{r}$ in terms of losses, maximum torque and weight, volume or inertia [23]. The size of the EVT, and thus its torque transmitting capabilities, are determined by the scaling factors and the DC bus voltage $V_{D C}$ (fixed value, defined within the battery unit).

The validated EVT model is considered scalable in the range of $\left[K_{a}, K_{r}\right] \in[0.8-2,0.8-2]$, the set [2,2] being equal
TABLE III

RATED \& SIMULATED POWERS AND TORQUES FOR THE EVT.

\begin{tabular}{l|ccc}
\hline & Rotor 1 & Rotor 2 & Stator \\
\hline$P_{\text {mech,rated }}[\mathrm{kW}]$ & 220 & 330 & - \\
$P_{\text {mech,sim }}[\mathrm{kW}]$ & 36 & 176 & - \\
\hline$T_{\max , \operatorname{rated}[\mathrm{Nm}]}$ & 470 & - & 705 \\
$T_{\max , \operatorname{sim}[\mathrm{Nm}]}$ & 158 & - & 520 \\
\hline
\end{tabular}

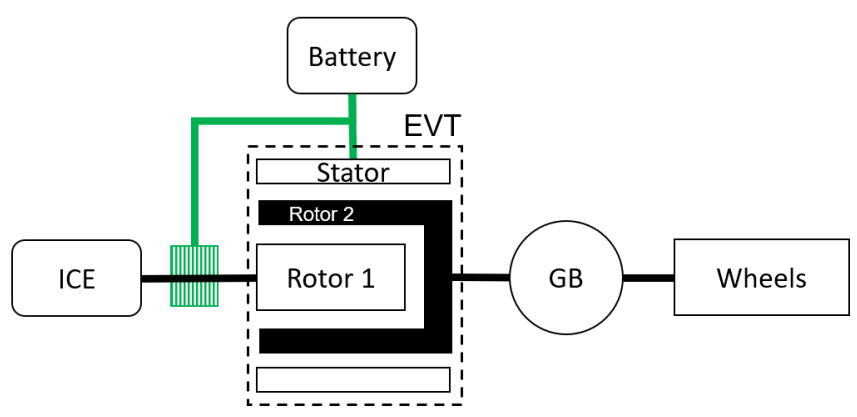

Fig. 3. EVT topology (power converters not shown).

to the largest achievable ratings. The EVT systems has been sized to $K_{a}=K_{r}=1.4$ to be able to complete the driving cycle and to reflect a similar material cost as the THS (see also Sec III-F. The resulting torque and power ratings (rated) as well as the maxima obtained during simulation $(\operatorname{sim})$ are shown in Table III

\section{E. Drivetrain modeling}

The forklift is modelled as a vehicle body with given mass (not including the hybrid drivetrain), a front area creating drag friction through a drag coefficient, tire friction through a road friction coefficient and translation-rotation mechanical conversion through a wheel radius and final drive ratio (and related efficiency). Table IV] summarizes the values for the Doosan D90s-7.

The ICE, EM and converter models are native to the toolbox and based on [13] and [24]. The battery, identical for THS and EVT topology, is modeled as a lossless constant voltage source with power limits determined by the maximum charge and discharge C-rate. The GB is also identical and is a shifted forward and backward system with an overall efficiency to account for the mechanical losses. Table $\mathrm{V}$ summarizes the values used to model the battery and GB for the simulation of both topologies.

The components are mathematically linked together to create the hybrid drivetrain topology model containing all the

TABLE IV

DoOSAN D90S-7 MODEL [20].

\begin{tabular}{c|c}
\hline Parameters & Doosan D90s-7 \\
\hline Mass [T] & 12275 \\
Wheels radius [m] & 0.498 \\
Wheel ratio [-] & 10 \\
Wheels efficiency [-] & 0.98 \\
Drag coefficient [-] & 0.45 \\
Rolling coefficient [-] & 0.01 \\
Front Area [ $\left.\mathrm{m}^{2}\right]$ & 21 \\
\hline
\end{tabular}


TABLE V

COMMON MODEL PARAMETERS.

\begin{tabular}{|c|c|}
\hline Parameter & Value \\
\hline Battery capacity [kWh] & 10 \\
\hline Battery voltage $[\mathrm{V}]$ & 600 \\
\hline Battery C-rate (charge, discharge) $\left[h^{-1}\right]$ & {$[5,10]$} \\
\hline $\begin{array}{c}\text { Gear ratio [-] } \\
\text { Gear efficiency [-] }\end{array}$ & 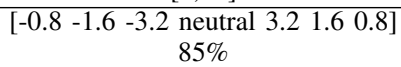 \\
\hline
\end{tabular}

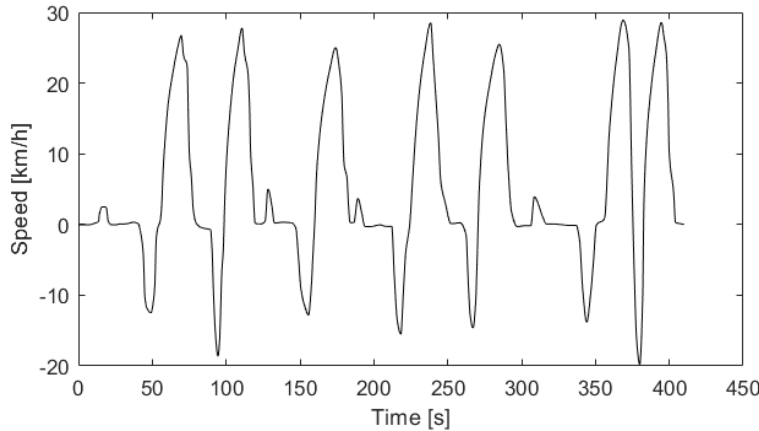

Fig. 4. Forklift driving cycle

system equations. By defining the input signals the topology is compiled into a causal model to be used in DP and the forward simulation.

The driving cycle is provided as a speed-time profile and will act as a boundary condition for DP. If the boundary condition cannot be met for each of the points in the profile i.e. there is no feasible set of inputs, the topology is not feasible for the given driving cycle. The chosen driving cycle is presented in Fig. 4] which is based on [25] and was adapted according to the mass ratio between the vehicle(s) studied in [25] and the forklift used in this paper.

\section{F. TCO analysis}

The two topologies are compared on a cost basis. The TCO sums the operational cost (based on the fuel consumption) and the capital costs (based on cost model for the relevant components). The capital cost includes the following components:

- EM: the cost model for electric motors takes into account the converter cost and material cost, where each has a fixed and variable part. The cost scales linearly with the rated power using a correlation from [26].

- EVT: The EVT is not an off-the-shelf component, so its material cost is estimated to be $150 \%$ of one EM (PMSM), as the EVT has two rotors but only one stator. The same coefficients and the same converter cost models are used as for the EM.

- GB: the cost model for the GB uses a cost per volume indicator where the volume is calculated according to the maximum gear ratio and torque.

- ICE: the cost for the ICE scales linearly with the rated power. The fixed cost are based on the fuel tank, the exhaust treatment and the start/stop system as from [26].
TABLE VI

USED COST PARAMETERS FOR THE TCO ANALYSIS.

\begin{tabular}{l|crl}
\hline & Fixed cost [€] & \multicolumn{3}{c}{ Variable cost [€/unit] } \\
\hline Inverter & 270 & 76 & $/ \mathrm{kW}$ \\
EM & 250 & 17.4 & $/ \mathrm{kW}$ \\
EVT & 375 & 26.1 & $/ \mathrm{kW}$ \\
GB & - & 1 & $/ 1$ \\
ICE & 1125 & 50 & $/ \mathrm{kW}$ \\
Battery & 300 & 0.42 & $/ \mathrm{Wh}$ \\
\hline
\end{tabular}

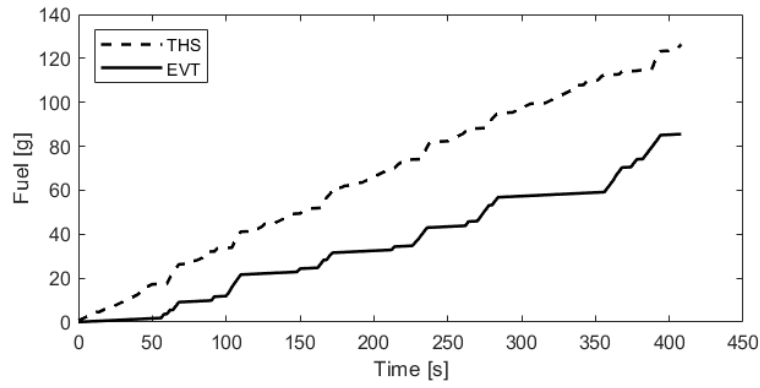

Fig. 5. Fuel consumption for THS and EVT topologies

- Battery: the cost model integrates a linear cost function of the open-loop voltage and capacitance of the battery.

Each used coefficient is listed in Table VI and based on a market study conducted by Flanders Make [27]. Additionally, the TCO analysis requires a total driven distance of the vehicle to calculate the total cost over the entire lifetime. Input from Flanders Make's industrial partners lead to a lifetime of 5 years and 600 operational hours per year, this totals to $25000 \mathrm{~km}$; each cycle is $952 \mathrm{~m}$.

\section{RESULTS AND DISCUSSION}

The DP toolbox makes sure that the optimal control inputs are used during the driving of one cycle. By calculating backwards all feasible ways of driving the cycle, it is ensured that the fuel consumption is minimal. The following paragraphs will discuss the outcome of this analysis.

\section{A. Fuel consumption}

Fig. 5 presents the fuel consumption over one driving cycle for the THS and EVT topologies. The THS consumes 126 grams of fuel while the EVT consumes 86 grams, which is an improvement of $32 \%$ for this off-highway application.

The fuel consumption for the EVT topology displays a less-linear trend (flatter pattern) than the THS topology. This implies a different usage of the ICE in each topology. Figs. $6 \mathrm{a}$ and $6 \mathrm{~b}$ show the torque versus speed plots on the ICE efficiency maps for the THS and EVT respectively. It is clear that the EVT topology allows to use the ICE in a more efficient zone compared to the THS topology. Furthermore, the ICE is either used in its best efficiency range or not used, whereas the THS configuration covers a larger working zone of the ICE thus lowering the average efficiency. Please note that the ICE model has been simplified to only idle (zero torque at any speed) and not stop (zero torque and zero 


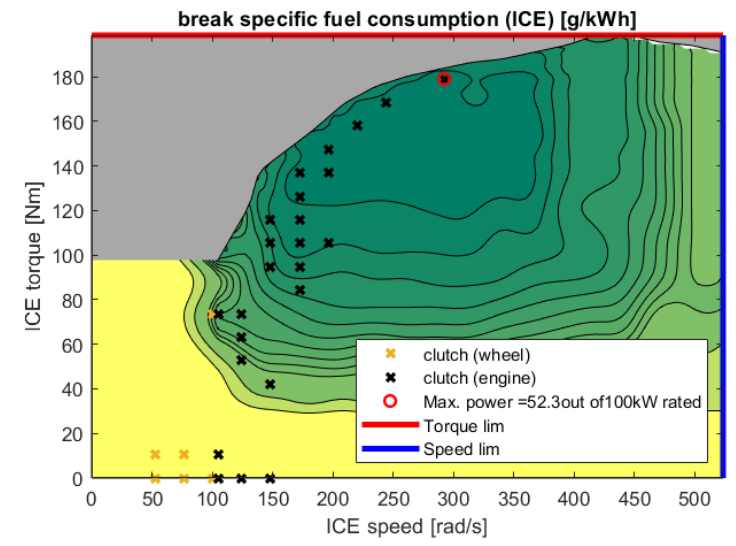

(a) THS

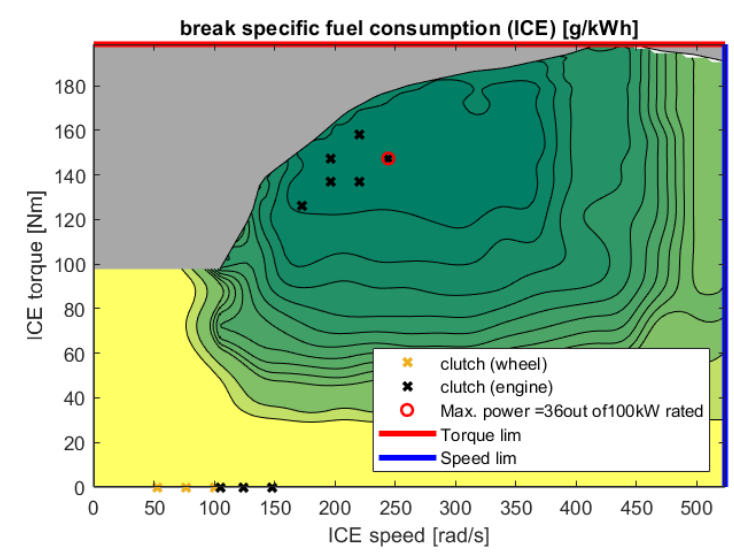

(b) EVT

Fig. 6. Torque versus speed plot of the entire driving cycle on the efficiency map of the ICE, for (a) THS system, (b) EVT system.

speed). The impact of this simplification is limited and does not favor any topology over the other.

The difference in behaviour can be explained using Fig. 7 , displaying the power flows of the ICE and battery, for each topology. The fuel consumption is placed on top of these power flows (on a different scale and right y-axis) to illustrate the fuel consumption of the ICE. Fig. $7 \mathrm{c}$ provides more insight in the acceleration and deceleration (= regenerating) phases.

Fig. 7a clearly depicts that the ICE and the battery split (almost in 50-50\% manner) the power required during the acceleration phases (positive power values) while the deceleration phases (negative power values) are handled purely by the battery. During these breaking phases, it is noticed that the ICE does not provide active power to the drivetrain (ICE power $=0$ ), meaning that the regenerative power produced is enough to balance the depletion of the battery during the acceleration phases. These curves correlate well with the torque-speed mapping of Fig. 6b and the flattened fuel consumption curve that was observed.

In comparison with the EVT topology, Fig. $7 \mathrm{~b}$ shows that the ICE and the battery are almost never driving the wheels simultaneously. This figure can be divided into three stages:

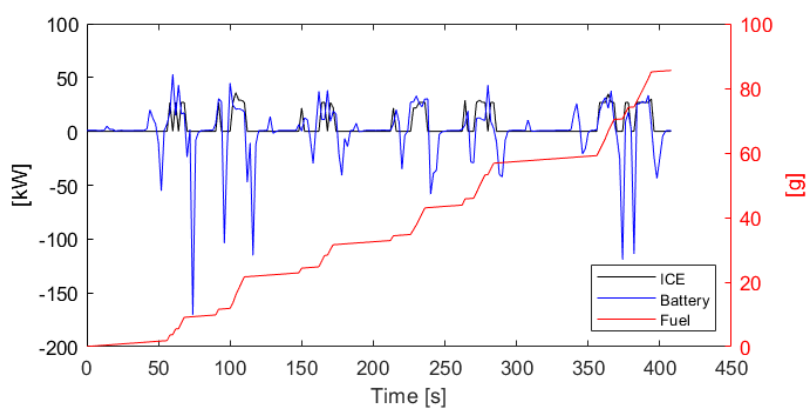

(a) Power flows for the ICE and the battery (left y-axis) \& Fuel consumption (right y-axis) for the EVT topology

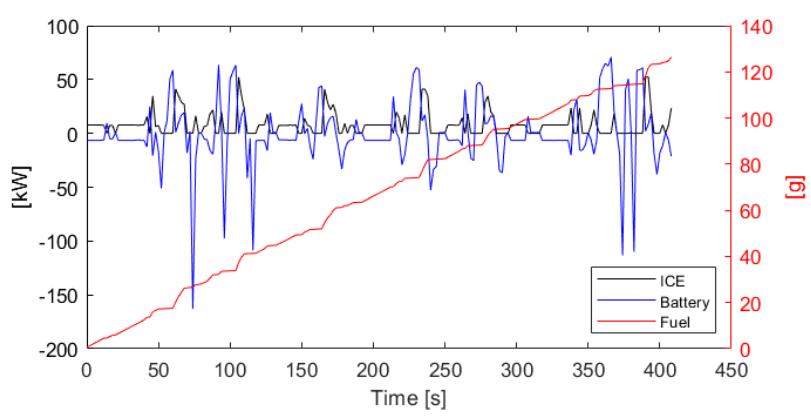

(b) Power flows for the ICE and the battery (left y-axis) \& Fuel consumption (right y-axis) for the THS topology

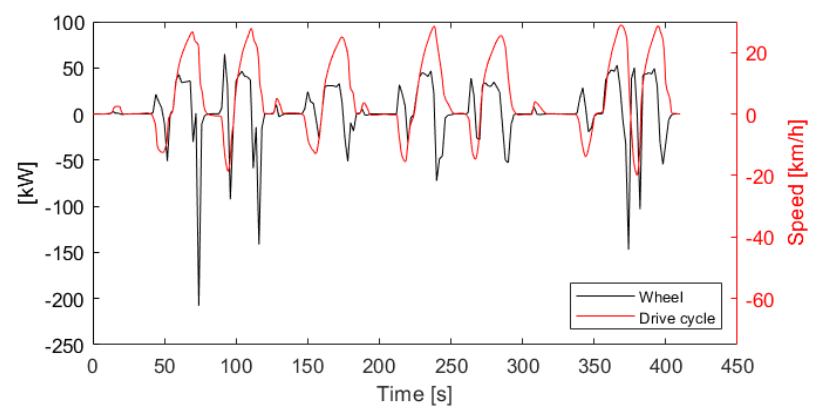

(c) Power to the wheel (for EVT, minimal difference for THS) and forklift driving cycle

Fig. 7. EVT 7a power flows, THS (7b) power flows and driving cycle 7c) power flows for the EVT topology

- "Steady-state" stage: mostly visible at the beginning of the driving cycle or between 300 and 350 seconds. No great acceleration or deceleration occurs at these moments. Nevertheless, it is noticeable that the ICE is providing power using EM2 which is directly absorbed by the battery (identical flows but reversed).

- Acceleration phase (power $>0$ ): a mismatch is notable between the power delivered by the battery and by the ICE. The increasing power of the battery is provided during the acceleration phases while the increasing power of the ICE is delivered just after it (in deceleration phases). The ICE is not able to brake the system, therefore, this implies that the ICE is used to charge the battery and that the DP process deems to be more efficient to drive the forklift using only electrical power 


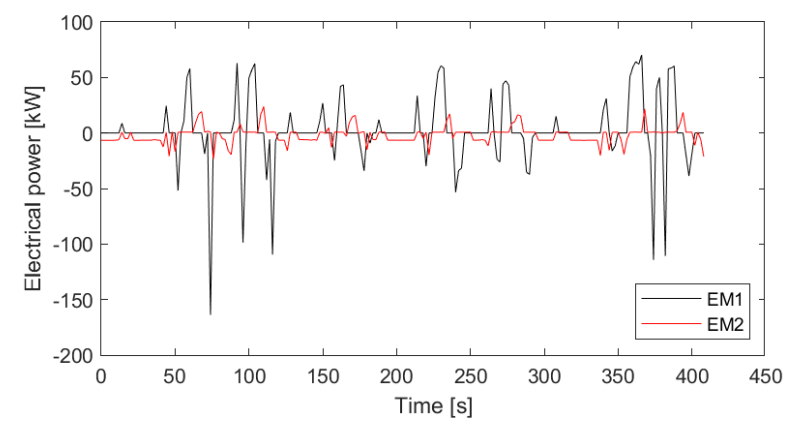

Fig. 8. Electrical power flows in EM1 and EM2.

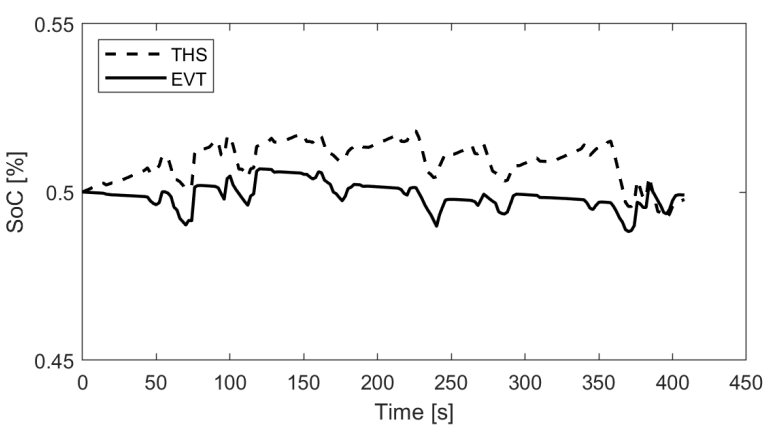

Fig. 9. SoC of the battery for THS and EVT topologies.

during peak demands.

- Deceleration phase (power $<0$ ): the power curves outline that the ICE usage can be lowered (or even stopped) during the regenerative period. The incoming power flow from the wheels is then handled by EM1 and EM2.

These statements are supported by Fig. 8 where the electrical power flows of EM1 and EM2 are presented.

\section{B. Battery ageing}

The battery state-of-charge (SoC) for the two topologies are presented in Fig. 9. These clearly show that no netelectrical consumption takes place. The THS SoC stands a bit higher than the EVT during the complete driving cycle, which can again be explained from the power profiles, as the battery in the THS topology is continuously being charged.

Given the way the batteries are used, the usage factor (UF), calculated as the amount of load variation in the battery times the lifetime of the vehicle $(25000 \mathrm{~km})$ and divided by the driven distance $(0.96 \mathrm{~km})$ and the number of expected cycles of the battery (10000), is lower for the EVT (43.7\%) than for the THS (58.4\%), which is a difference of $25 \%$. The capital cost of the batteries remains the same as the UF is $<100 \%$ for both topologies and thus the lifetime of one battery is sufficient for the considered lifetime of the vehicle. However, if the lifetime would be longer, the battery in the EVT topology would thus last longer.

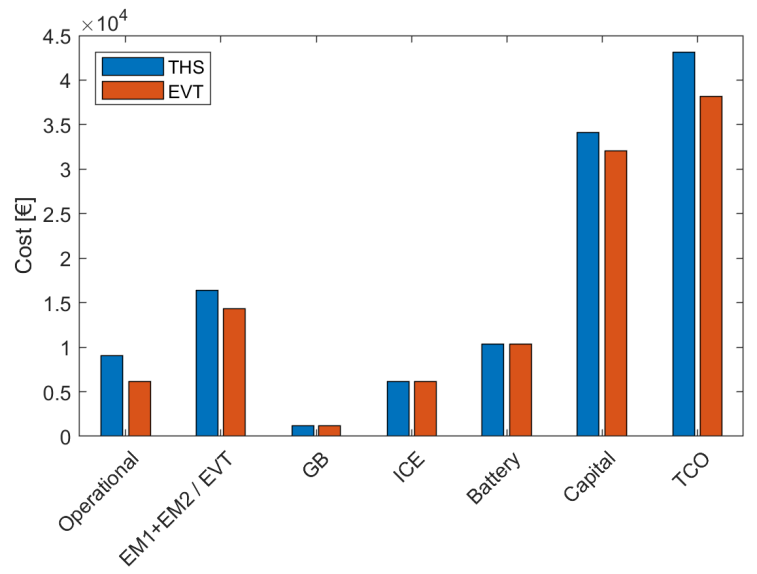

Fig. 10. TCO analysis.

TABLE VII

THS AND EVT COMPARISON.

\begin{tabular}{l|ccc}
\hline & THS & EVT & $\Delta$ \\
\hline Fuel [g] & 126.39 & 85.54 & $-32 \%$ \\
Battery usage [\% of lifetime] & 58.4 & 43.7 & $-25 \%$ \\
Capital cost of transmission [€] & 34080 & 32032 & $-6 \%$ \\
Lifetime fuel cost [€] & 9085 & 6149 & $-32 \%$ \\
TCO [€] & 43165 & 38181 & $-12 \%$ \\
\hline
\end{tabular}

\section{TCO analysis}

A full TCO analysis of the topologies is given in Fig. 10. The gain in cost is achieved by the decreased operational cost and decreased capital cost of the EVT. The gain in cost is $11.5 \%$ (decreased from $€ 43165$ to $€ 38181$ ).

\section{CONCLUSIONS}

This paper shows the potential of integrating an electrical variable transmission (EVT) to electrify an off-highway drivetrain. The EVT combines the functionality of a motor, a generator and a variable transmission, which are key elements in a hybrid drivetrain. It was benchmarked against the Toyota Hybrid System (THS) and evaluated with Dynamic Programming (DP) to ensure a global optimal control strategy. Table VII summarizes the results.

The fuel consumption is reduced by roughly $30 \%$ while the overall cost of the transmission, primarily based on the material cost, is similar. The detailed power flow analysis illustrates that the EVT is more flexible in decoupling the ICE from the wheels, which leads to an overall higher efficiency for the ICE. Furthermore, the EVT relies less on the battery to store energy for later usage, which results in a better ageing situation (in this case a $25 \%$ improvement).

Overall, the analyses of the two drivetrains show that the total cost of ownership for a Doosan D90s-7 forklift has reduced by roughly $10 \%$. As a transmission with an electrical port, the EVT offers significant advantages for electrification.

\section{REFERENCES}

[1] A. M. El-Refaie, "Motors/generators for traction/propulsion applications: A review," IEEE Vehicular Technology Magazine, vol. 8, no. 1, pp. 90-99, March 2013. 
[2] M. Hoeijmakers, "Rotating electromechnical converter," 2015, US 9 018 863, DOI US 2010/0311130 Al.

[3] F. Verbelen, "A comparative study of mechanical and electrical variable transmissions," Ph.D. dissertation, Ghent University, 2019.

[4] J. Gao, J. Song, and Y. Du, "Mode and control strategy of HEV equipped with EVT," in 2009 IEEE Vehicle Power and Propulsion Conference, Sep. 2009, pp. 1822-1825.

[5] A. A. Abdelsalam and S. Cui, "A fuzzy logic global power management strategy for hybrid electric vehicles based on a permanent magnet electric variable transmission," Energies, vol. 5, no. 4, pp. 1175-1198, 2012.

[6] Q. Xu, S. Cui, L. Song, and Q. Zhang, "Research on the power management strategy of hybrid electric vehicles based on electric variable transmissions," Energies, vol. 7, no. 2, pp. 934-960, 2014.

[7] E. Vinot, R. Trigui, Y. Cheng, C. Espanet, A. Bouscayrol, and V. Reinbold, "Improvement of an EVT-based HEV using dynamic programming," IEEE Trans. on Vehicular Technology, vol. 63, no. 1, pp. 40-50, Jan 2014.

[8] F. Verbelen, H. Vansompel, A. Abdallh, K. Stockman, and P. Sergeant, "Design methodology for a PM electrical variable transmission used in HEV," in Energy Efficiency in Motor Driven Systems (EEMODS), 2019.

[9] M. Vafacipour, M. E. Baghdadi, F. Verbelen, P. Sergeant, J. Van Mierlo, K. Stockman, and O. Hegazy, "Technical assessment of utilizing an electrical variable transmission system in hybrid electric vehicles," in 2018 IEEE Transportation Electrification Conference and Expo, AsiaPacific, June 2018, pp. 1-5.

[10] S. Vanhee, "Concept study of an electrical variable transmission for off-highway applications," Master's thesis, Ghent University, 2015.

[11] Y. Madrane, "Feasibility analysis of electrical variable transmission in off-highway vehicle drivetrains: Fork-lift as a case study," Master's thesis, Joint program between ULB University \& VUB University, Brussels, Belgium, 2019.

[12] S. Seyed Hosseini, "Case study: Toyota hybrid synergy drive," Available online at www.researchgate.net Accessed: March 1, 2020.

[13] J. Goos, C. Criens, and M. Witters, "Automatic evaluation and optimization of generic hybrid vehicle topologies using dynamic programming," in 20th IFAC World Congress, vol. 50, 2017.

[14] E. Nordlund and S. Eriksson, "Test and verification of a four-quadrant transducer for HEV applications," 2005 IEEE Vehicle Power and Propulsion Conference, vol. 2005, no. December, pp. 37-41, 2005.

[15] J. Druant, H. Vansompel, F. De Belie, J. Melkebeek, and P. Sergeant, "Torque analysis on a double rotor electrical variable transmission with hybrid excitation," IEEE Trans. on Industrial Electronics, vol. 64, no. 1, pp. 60-68, 2017.

[16] L. S. Pontriagin, V. G. Boltyanskie, R. V. Gamkrelidze, and E. F. Miŝenko, The Mathematical Theory of Optimal Processes. Interscience Publishers, 1962.

[17] H. P. Geering, Optimal Control with Engineering Applications. Springer Science \& Business Media, 2007.

[18] D. Bertsekas, Dynamic Programming and Optimal Control, ser. Athena Scientific optimization and computation series. Athena Scientific, 2012, no. v. 1.

[19] K. v. Berkel, B. d. Jager, T. Hofman, and M. Steinbuch, "Implementation of dynamic programming for optimal control problems with continuous states," IEEE Trans. on Control Systems Technology, vol. 23, no. 3, pp. 1172-1179, 2015.

[20] Doosan, "Doosan d90s-7 specifications \& technical data (2017-2019)," Available online at www.lectura-specs.com Accessed: March 1, 2020

[21] J. Druant, H. Vansompel, F. De Belie, and P. Sergeant, "Loss Identification in a double rotor electrical variable transmission," IEEE Trans. on Industrial Electronics, vol. 64, no. 10, pp. 7731-7740, 2017.

[22] F. Verbelen, A. Abdallh, H. Vansompel, K. Stockman, and P. Sergeant, "Sizing methodology based on scaling laws for a permanent magnet

[24] A. Abdallh and M. Witters, "Optimal control and design of hybrid electric vehicles including bi-directional DC-DC converters," in 2017 IEEE Vehicle Power and Propulsion Conference (VPPC), Dec 2017, pp. 1-5. electrical variable transmission," IEEE Trans. on Industrial Electronics, vol. 67, no. 3, pp. 1739-1749, March 2020.

[23] S. Stipetic and D. Zarko, "Scaling laws for synchronous permanent magnet machines," in Eleventh International Conference on Ecological Vehicles and Renewable Energies (EVER), 2015, pp. 1-7.

[25] S. Mercati, F. Panizzolo, and G. Profumo, "Power split hydromechanical variable transmission (HVT) for off-highway application," 10th International Fluid Power Conference, 2016.

[26] M. Friesa, M. Kerlera, S. Rohra, S. Schickram, M. Sinning, and M. Lienkamp, "An overview of costs for vehicle components, fuels, greenhouse gas emissions and total cost of ownership update 2017," Garching, Germany, 2017. Available online at https://steps.ucdavis.edu Accessed: March 1, 2020.

[27] B. Lenaerts, A. Abdallh, D. Maes, B. Mrak, T. Galle, and W. De Waele, "Total cost of ownership optimization of manufacturing machines with fast energy storage," in 2018 IEEE 18th Intern. Power Electronics and Motion Control Conference, Aug 2018, pp. 784-789.

\section{BIOGRAPHIES}

Thomas Vandenhove was born in Brussels, Belgium, in 1996. He received the B.Sc. and M.Sc. (H.O.C.) degree in 2017 and 2019 respectively in Automation Engineering from ECAM - Brussels Engineering School. He is currently an Associate Application Engineer within the MotionS Corelab of Flanders Make. His current research interests are power electronics, control, hybrid drivetrains and electrical grid to machine couplings.

Ahmed A-E. Abdallh (M'12) received the Ph.D. degree in electromechanical engineering from Ghent University in 2012, followed by a three-years postdoctoral researcher at the same institute. From 2015 to 2019, he was working as a Research Engineer at Flanders Make (the strategic research centre for the manufacturing industry in Belgium). Currently, he is working with ArcelorMittal global R\&D in Gent as a research engineer, where he is conducting a research on electromagnetic applications of electrical steels in different industrial domains. His research interests include electric machines design and electrification of industrial/vehicle drivetrains.

This research was performed while Ahmed was working at Flanders Make.

Florian Verbelen was born in Kortrijk, Belgium, in 1990. He received the Master's degree in electro mechanical engineering from the Technical University College of West Flanders, Kortrijk, Belgium, in 2013. In 2019, he obtained the Ph.D. degree at Ghent University where he worked on the energetic and dynamic impact of variable transmissions on advanced drivetrains. He is currently a Postdoctoral Researcher with Ghent University. His current research interests focus on variable transmissions and the impact of low level motor control on torque ripple and efficiency.

Mats Vande Cavey is a research engineer at Flanders Make vzw in Belgium working in the DecisionS core lab of Flanders Make since 2016. After receiving his M.Sc. in Energy Engineering at the KU Leuven, Belgium in 2012 Mats joined the Applied Mechanics and Energy Conversion division (TME) at the Mechanical Engineering department at KU Leuven where he worked on computation tools and model predictive control for energy management and building systems simulation also focusing on integrating industrial energy storage and usage with renewable energy. Currently Mats is working on control, modelling and automated design of energy systems in the manufacturing industry.

Jeroen Stuyts was born in 1990 in Belgium. He received the B.Sc., M.Sc. and $\mathrm{PhD}$ degree in 2011, 2013 and 2018 respectively as Energy and Electrical Engineer from the KU Leuven, Belgium, with a doctoral thesis on "Implementation of Unbalance Compensation using Grid-supporting Converters". His research interests are power electronics, drives, industrial machines, renewable energy sources and their grid coupling. He is currently working at Flanders Make as Technology Domain Leader on Single Drivetrains within the MotionS corelab. 